\title{
Inhibition of miR-96 enhances the sensitivity of colorectal cancer cells to oxaliplatin by targeting TPM1
}

\author{
TINGRUI GE, PING XIANG, HAIBING MAO, SHUMIN TANG, JINYI ZHOU and YONGGANG ZHANG
}

\begin{abstract}
Department of Colorectal Surgery, The First People's Hospital of Lianyungang, Lianyungang, Jiangsu 222002, P.R. China
\end{abstract}
Received June 26, 2019; Accepted February 20, 2020

DOI: $10.3892 /$ etm.2020.8936

\begin{abstract}
Colorectal cancer (CRC) is one of the major threats to human health worldwide. In the treatment of CRC, chemoresistance affects the efficacy of platinum-based therapies. Oxaliplatin is one of the most commonly used first-line medications for the treatment of CRC; however, chemoresistance is common among patients receiving oxaliplatin treatment, which significantly decreases its therapeutic efficacy. The present study focused on the roles of microRNA (miR)-96 in the oxaliplatin resistance of CRC cells and the underlying mechanisms. First, the expression of miR-96 was compared between CRC and adjacent tissues. Furthermore, target genes of miR-96 were predicted, and a dual-luciferase reporter assay was employed to confirm whether the candidate tropomyosin 1 (TPM1) is a direct target of miR-96. In addition, CRC cells were transfected with miR-96 inhibitor, miR-negative control, small interfering RNA (siRNA) targeting TPM1 or siRNA NC, and then treated with oxaliplatin. CCK-8 assay and flow cytometry were performed to examine the proliferation and apoptosis of the CRC cell line SW480. Next, reverse transcription-quantitative PCR and western blot analysis were performed to determine the mRNA and/or protein levels of miR-96, Bcl-2, BAX and TPM1. The results indicated that miR-96 was upregulated in CRC compared with normal adjacent tissues, while TPM1 was downregulated. The luciferase activity was reduced following transfection with miR-96 mimics and luciferase reporter plasmid containing the wild-type sequence of the 3'-untranslated region of TPM1. Furthermore, knockdown of miR-96 combined with oxaliplatin reduced the viability and induced apoptosis of CRC cells, which was further verified by decreased expression of $\mathrm{Bcl}-2$ and the increased expression of TPM1 and BAX. Taken together, the downregulation of miR-96 enhanced the sensitivity of CRC cells to oxaliplatin.
\end{abstract}

Correspondence to: Dr Yonggang Zhang, Department of Colorectal Surgery, The First People's Hospital of Lianyungang, 182 North Tongguan Road, Lianyungang, Jiangsu 222002, P.R. China E-mail: qiangtang96@163.com

Key words: microRNA-96, tropomyosin 1, oxaliplatin, chemosensitivity, colorectal cancer

\section{Introduction}

Colorectal cancer (CRC) is one of the most common malignancies worldwide $(1,2)$. The routine therapeutic strategy for CRC is surgery combined with platinum-based treatment $(3,4)$. Oxaliplatin, as a platinum-based drug, inhibits the progression of CRC by binding to and cross-linking the DNA of cancer cells (3). Although advances have been made in the treatment of CRC, the overall survival rate of CRC patients remains unsatisfactory due to chemoresistance and recurrence $(5,6)$. A previous study indicated that chemoresistance emerges in over 20\% of all CRC patients within 6 months of therapy (7). Therefore, exploration of novel therapies to enhance the chemosensitivity of CRC cells to oxaliplatin is imperative.

MicroRNAs (miRNAs/miRs) are a class of non-coding RNAs. Increasing evidence demonstrated that miRNAs are involved in various physiological and pathological processes, including cell proliferation, metastasis, invasion and apoptosis, and dysregulation of certain miRNAs is associated with multiple types of cancer by acting as either oncogenic or tumor-suppressive agents (8-12). miR-96 plays a vital role in the progression of various cancer types $(13,14)$. Studies indicated that miR-96 has a role as an oncogene or tumor suppressor, depending on the type of cancer (13-16). In CRC, miR-96 demonstrated to act as an oncogene and increase cell proliferation $(17,18)$. However, the potential function of miR-96 in regulating the sensitivity of CRC to oxaliplatin and the associated mechanisms remain to be elucidated.

In the present study, the possible role of miR-96 in regulating the resistance of CRC cells to oxaliplatin was investigated. The proliferation and apoptotic rates of CRC cells treated with oxaliplatin and miR-96 inhibitor was determined. Subsequently, the expression of proliferation- and apoptosis-associated genes was examined after treatment with oxaliplatin and miR-96 inhibitor. The results indicated that knockdown of miR-96 enhances the sensitivity of CRC cells to oxaliplatin.

\section{Materials and methods}

Tissue samples. A total of $40 \mathrm{CRC}$ tissues and 40 adjacent tumor tissues were obtained from 40 patients ( 25 males and 15 females; age range 48-74 years) diagnosed with CRC at the First People's Hospital of Lianyungang (China) between Jan 2016 and Oct 2017. The tissue samples were confirmed by pathological examination and immediately stored in liquid 
nitrogen after surgery. Tumor size was recorded as the diameter of the tumor; metastasis was confirmed as positive when tumor cells were found on the nearby lymph nodes and the degree of differentiation of the tumor was determined by histological examination. All patients provided written informed consent. The present study was approved by the Ethics Committee of the First People's Hospital of Lianyungang (China).

Cell culture and transfection. The CRC cell line SW480 was purchased from the American Type Culture Collection. Cells were cultured in RPMI-1640 medium (Gibco; Thermo Fisher Scientific, Inc.) containing 10\% FBS (Gibco; Thermo Fisher Scientific, Inc.) and $1 \%$ penicillin/streptomycin (Gibco; Thermo Fisher Scientific, Inc.) at $35^{\circ} \mathrm{C}$ in an atmosphere with $5 \% \mathrm{CO}_{2}$. miR-96 inhibitor, miR-negative control (miR-NC), small interfering RNA (siRNA) targeting tropomyosin 1 (TPM1) and siRNA NC were designed and provided by Guangzhou RiboBio Co., Ltd. The cells were transfected with miR-96 inhibitor, miR-NC, miR-96 mimics, siRNA TPM1 and siRNA NC using Lipofectamine ${ }^{\mathrm{TM}} 3000$ (Invitrogen; Thermo Fisher Scientific, Inc.) for $48 \mathrm{~h}$. A total of $20 \mu \mathrm{mol} / 1$ oxaliplatin (Sigma-Aldrich; Merck KGaA) was used to investigate oxaliplatin sensitivity. The sequences of the miRNAs and siRNAs used were: miR-96 inhibitor, 5'-GCAAAAAUGUGCUAG UGCCAAA-3'; miR-NC, 5'-CAGUACUUUUGUGUAGUA CAA-3'; miR-96 mimic, 5'-UUUGGCACUAGCACAUUU UUGC-3', siRNA TPM1 5'-CCCGTAAGCTGGTCATCAT-3' and siRNA NC, 5'-CCCAACGGTTGACTGTCAT-3'.

CCK-8 assay. Cells were seeded into a 96-well plate at a density of $2 \times 10^{3}$ cells/well. At $0,8,16$ and $44 \mathrm{~h}$, the cells were stained with $20 \mu \mathrm{l}$ CCK-8 solution (Beyotime Institute of Biotechnology) for $4 \mathrm{~h}$ according to the manufacturer's instructions. Subsequently, the absorbance rate of the cells at a wavelength of $450 \mathrm{~nm}$ was examined with a spectrophotometer (Thermo Fisher Scientific, Inc.).

Flow cytometry. Cells were trypsinized and collected by centrifugation at room temperature at $1,000 \times \mathrm{g}$ for $5 \mathrm{~min}$. The cells were then washed with pre-cooled PBS and resuspended in binding buffer (Beyotime Institute of Biotechnology) in the centrifuge tubes. Subsequently, the cells were stained with Annexin-V FITC and propidium iodide (BD Biosciences). Finally, the apoptotic rate of SW480 cells was determined with a flow cytometer (EPICS XL; Beckman Coulter) and analyzed using FlowJo software (version 10.5.3; FlowJo LLC).

Reverse transcription-quantitative PCR (RT-qPCR). Cells were lysed with TRIzol ${ }^{\circledR}$ reagent (Invitrogen; Thermo Fisher Scientific, Inc.) and total RNA was extracted according to the manufacturer's instructions. RNA was reverse transcribed to generate cDNA with the PrimeScript RT Reagent kit (Takara Bio, Inc.). Subsequently, qPCR was performed on an ABI Prism 7500 Real-Time PCR system (Thermo Fisher Scientific, Inc.) using the SYBR ${ }^{\text {TM }}$ Green PCR Master Mix (cat. no. 431270; Applied Biosystems; Thermo Fisher Scientific, Inc.). The following thermocycling conditions were used: Initial denaturation at $95^{\circ} \mathrm{C}$ for $10 \mathrm{~min}$; followed by 40 cycles of $95^{\circ} \mathrm{C}$ for $15 \mathrm{sec}$ and $60^{\circ} \mathrm{C}$ for $30 \mathrm{sec}$. The relative RNA expression was determined using the $2^{-\Delta \Delta \mathrm{Cq}}$ method (19). U6 and GAPDH served as the internal reference gene for miRNA and mRNA, respectively. Each experiment was performed in triplicate. The following primer pairs were used for the qPCR: miR-96 forward, 5'-TTT GGCACTAGCACAT-3' and reverse, 5'-GAGCAGGCTGGA GAA-3'; U6 forward, 5'-ATTGGAACGATACAGAGAAGA T-3' and reverse, 5'-GGAACGCTTCACGAATTT-3'; TPM1 forward, 5'-CTCTCAACGATATGACTTCCA-3' and reverse, 5'-TTTTTTTAGCTTACACAGTGTT-3'; Bcl-2 forward, 5'-TTC TTTGAGTTCGGTGGGGTC-3' and reverse, 5'-TGCATATTT GTTTGGGGCAGG-3'; BAX forward, 5'-TCCACCAAGAAG CTGAGCGAG-3' and reverse, 5'-GTCCAGCCCATGATGGTT CT-3'; GAPDH forward, 5'-CGAGCCACATCGCTCAGACA-3' and reverse, 5'-GTGGTGAAGACGCCAGTGGA-3'.

Western blotting. Cells or tissues were lysed and total protein was extracted using RIPA buffer (Sigma-Aldrich; Merck $\mathrm{KGaA}$ ). Protein concentration was measured with a bicinchoninic acid kit (Pierce; Thermo Fisher Scientific, Inc.). Subsequently, $30 \mu \mathrm{g}$ of protein/lane was separated by $10 \%$ SDS-PAGE and transferred onto PVDF membranes. The membranes were blocked with $5 \%$ non-fat milk for $1 \mathrm{~h}$ at $37^{\circ} \mathrm{C}$ under exclusion of direct light. Subsequently, the membranes were incubated with the following primary antibodies (all purchased from Abcam) overnight at $4^{\circ} \mathrm{C}$ : Anti-Bcl-2 (cat. no. ab59348; 1:1,000), anti-BAX (cat. no. ab32503; 1:1,000), anti-TPM1 (cat. no. ab55915; 1:1,000) and anti-GAPDH (cat. no. ab9485; 1:2,000). The membranes were then incubated with horseradish peroxidase-labeled secondary antibodies (cat. no. ab6721; 1:5,000; Abcam) at $37^{\circ} \mathrm{C}$ for $1 \mathrm{~h}$. Protein signal was visualized with an ECL detection system (Thermo Fisher Scientific, Inc.) and the density of the protein bands was evaluated with a sensitive chemiluminescent substrate (Sigma-Aldrich; Merck KGaA). GAPDH was used as an internal control. Densitometric analysis was performed using Image J (version 2.1.4; National Institutes of Health).

Dual-luciferase reporter assay. The online database TargetScan (http://www.targetscan.org/vert_71/) predicted TPM1 as a target gene of miR-96. The wild-type or mutant sequence from the 3'-untranslated region (3'-UTR) of TPM1 containing the binding site for miR-96 was cloned into the luciferase gene reporter vector pMIR (Thermo Fisher Scientific, Inc.). The reporter plasmids were then co-transfected with miR-96 mimics or miR-NC into SW480 cells with Lipofectamine ${ }^{\circledR} 2000$ transfection reagent for $48 \mathrm{~h}$ (Invitrogen; Thermo Fisher Scientific, Inc.). A Dual-Lumi ${ }^{\mathrm{TM}}$ Dual Luciferase Reporter Assay kit (Beyotime Institute of Biotechnology) was used to detect luciferase activity on a GloMax Explorer Multimode microplate reader (Promega Corp.). Luciferase activity was normalized to Renilla luciferase activity.

Statistical analysis. SPSS 18.0 (SPSS, Inc.) was used to analyze the data. Values are expressed as the mean \pm SD. Paired Student's t-test was performed to compare the expression of miR-96 and TPM1 in CRC tissues and adjacent normal tissues. One-way ANOVA with Tukey's post hoc test or two-way ANOVA with Sidak's multiple comparisons test was applied to analyze differences among multiple groups. The $\chi^{2}$ test was used for the analysis of clinical information presented in Table I. Pearson's correlation coefficient was used 
Table I. Clinical information of patients.

miR-96 expression

\begin{tabular}{|c|c|c|c|}
\hline \multirow[b]{2}{*}{ Parameters } & \multicolumn{2}{|c|}{ miR-96 expression } & \multirow[b]{2}{*}{ P-value } \\
\hline & $\begin{array}{l}\text { High (relative expression of miR-96 in CRC } \\
\text { tissue }>1 ; n=25 \text { ) }\end{array}$ & $\begin{array}{l}\text { Low (relative expression of miR-96 in } \\
\text { CRC tissue }<1 ; n=15 \text { ) }\end{array}$ & \\
\hline Sex & & & 0.4638 \\
\hline Male & 17 & 8 & \\
\hline Female & 8 & 7 & \\
\hline Age & & & 0.8003 \\
\hline$<60$ & 16 & 9 & \\
\hline$\geq 60$ & 9 & 6 & \\
\hline Tumor size $(\mathrm{cm})$ & & & $0.0258^{\mathrm{a}}$ \\
\hline$\geq 5$ & 14 & 3 & \\
\hline$<5$ & 11 & 12 & \\
\hline Differentiation & & & 0.4119 \\
\hline Well-moderate & 10 & 8 & \\
\hline Poor & 15 & 7 & \\
\hline Metastasis & & & $0.0222^{\mathrm{a}}$ \\
\hline Negative & 9 & 11 & \\
\hline Positive & 16 & 4 & \\
\hline
\end{tabular}

${ }^{\mathrm{a}} \mathrm{P}<0.05$. miR-96, microRNA-96; CRC, colorectal cancer.

for correlation analysis. $\mathrm{P}<0.05$ was considered to indicate a statistically significant difference.

\section{Results}

miR-96 is upregulated in CRC tissues. RT-qPCR was performed to determine the expression of miR-96 in CRC tissues and normal tissues. The results indicated that the expression level of miR-96 in CRC tissues was significantly higher compared with adjacent tissues (Fig. 1). Moreover, increased expression of miR-96 was positively associated with the tumor size and metastasis (Table I).

TPM1 is a direct target of miR-96. The online database TargetScan predicted that miR-96 directly binds to the 3'-UTR of TPM1 (Fig. 2A). The expression level of TPM1 in CRC tissues was significantly lower compared with adjacent tissues (Fig. 2B), and the expression of miR-96 and TPM1 was negatively correlated ( $\mathrm{r}=-0.4459$; $\mathrm{P}=0.0039$; Fig. $2 \mathrm{C}$ ). To further confirm the targeting interaction, a dual-luciferase reporter assay was performed. The results demonstrated that the relative luciferase activity of CRC cells transfected with miR-96 mimics and a luciferase reporter vector containing the wild-type sequence of the 3'-UTR of TPM1 was significantly decreased compared with the NC group, while there were no significant differences between miR-96 mimic- and NC-transfected cells in the mutant group (Fig. 2D).

Downregulation of miR-96 increases the sensitivity of $C R C$ cells to oxaliplatin via targeting TPM1. Cells were transfected with miR-96 inhibitor and a CCK-8 assay and flow

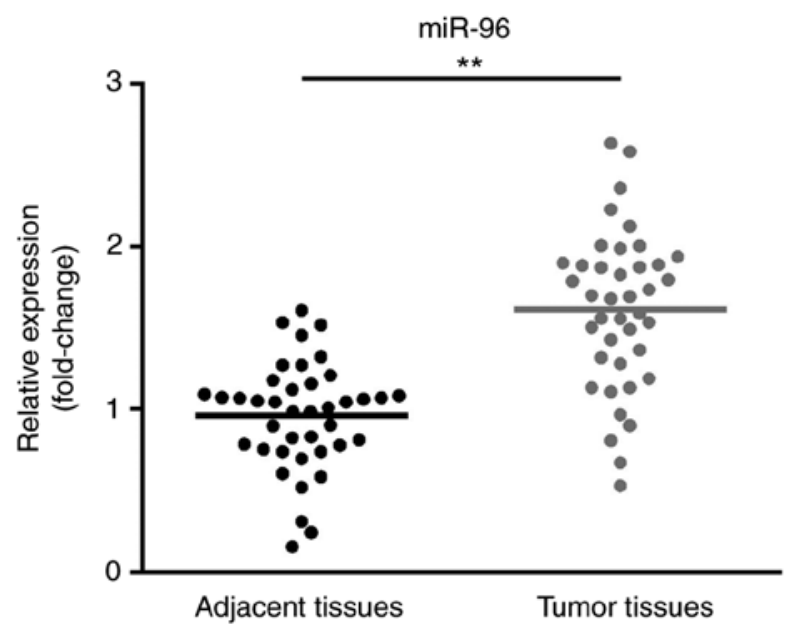

Figure 1. Expression of miR-96 in CRC tissues. The expression of miR-96 in CRC tissues was significantly higher compared with adjacent normal tissues. ${ }^{* *} \mathrm{P}<0.01$. miR-96, microRNA-96; CRC, colorectal cancer.

cytometry were performed to determine the cell viability and apoptosis of CRC cells following different treatments. As presented in Fig. 3, the expression of miR-96 was significantly downregulated following transfection with miR-96 inhibitor compared with the miR-NC group (Fig. 3A), and the expression of TPM1 was significantly downregulated following transfection with siRNA-TPM1 compared with the siRNA-NC group (Fig. 3B), suggesting that TPM1 knockdown was successfully performed. Furthermore, the results of the CCK- 8 assay indicated that oxaliplatin significantly 
A
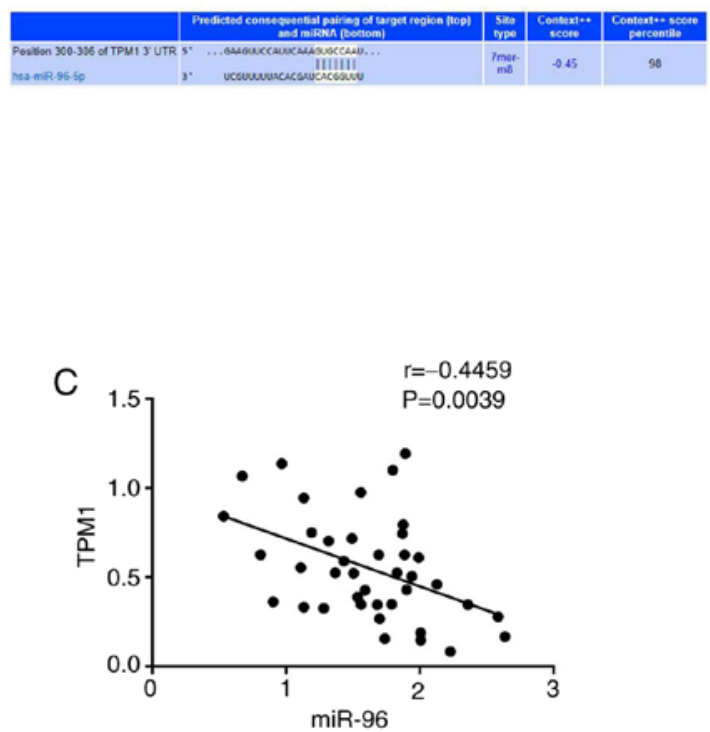

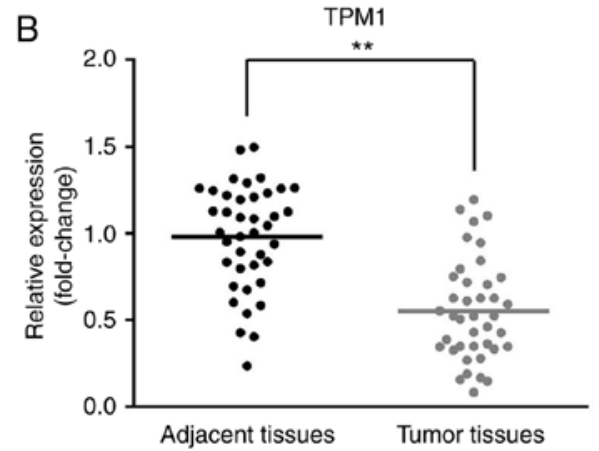

D

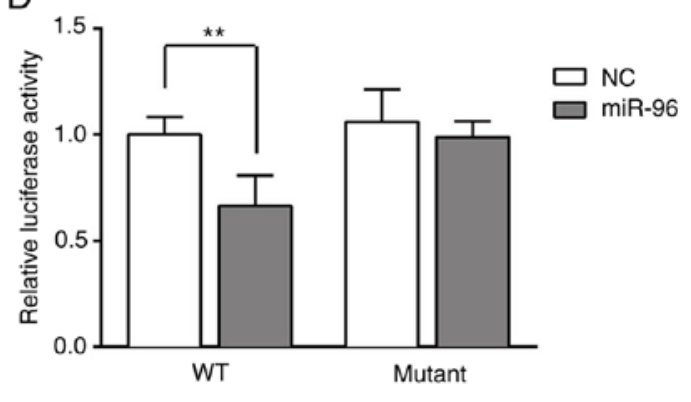

Figure 2. TPM1 is directly targeted by miR-96. (A) TargetScan predicted TPM1 as a target of miR-96. (B) The expression of TPM1 in CRC tissues was significantly lower compared with adjacent tissues. (C) Correlation between the expression of miR-96 and TPM1 in CRC tissues. (D) In cells containing the WT-TPM1 3'-UTR, the relative luciferase activity of SW480 significantly decreased following transfection with miR-96 mimics compared with the NC group. No significant difference was found between miR-96 mimic- and NC-transfected groups containing mutant TPM1 3'-UTR showed. ${ }^{* *} \mathrm{P}<0.01$. CRC, colorectal cancer; 3'-UTR, 3'-untranslated region; TPM1, tropomysin 1; miR-96, microRNA-96; WT, wild-type; NC, negative control.

inhibited the viability of SW480 cells compared with the blank control group, and the cell viability was markedly decreased in the oxaliplatin + miR-96 inhibitor group compared with the oxaliplatin group (Fig. 4A). In addition, the apoptotic rate of the SW480 cells in the oxaliplatin group was significantly higher compared with the control group, and the apoptotic rate in the oxaliplatin + miR-96 inhibitor group significantly increased compared with the oxaliplatin group (Fig. 4B). Transfection of siRNA TPM1 in oxaliplatin + miR-96 inhibitor-treated cells significantly decreased cell viability and increased cell apoptosis compared with the oxaliplatin + miR-96 inhibitor group (Fig. 4).

Inhibition of miR-96 decreases the expression of Bcl-2 and $B A X$, and increases expression of TPM1. As presented in Fig. 5, oxaliplatin significantly decreased the mRNA and protein expression of $\mathrm{Bcl}-2$, and increased the expression of TPM1 and BAX compared with the control group. Changes in protein expression were more apparent following treatment with oxaliplatin + miR-96 inhibitor and were attenuated in cells co-transfected with siRNA TPM1.

\section{Discussion}

Although advances have been made in the treatment of CRC in recent years (20), multidrug resistance remains the major obstacle of chemotherapy (3). Aberrant expression of certain miRNAs has crucial roles in the development of chemoresistance (21). The tumor suppressor gene $m i R-410$ is downregulated in lung cancer tissues and induced apoptosis in lung cancer cells (22). miR-25 is downregulated in melanoma
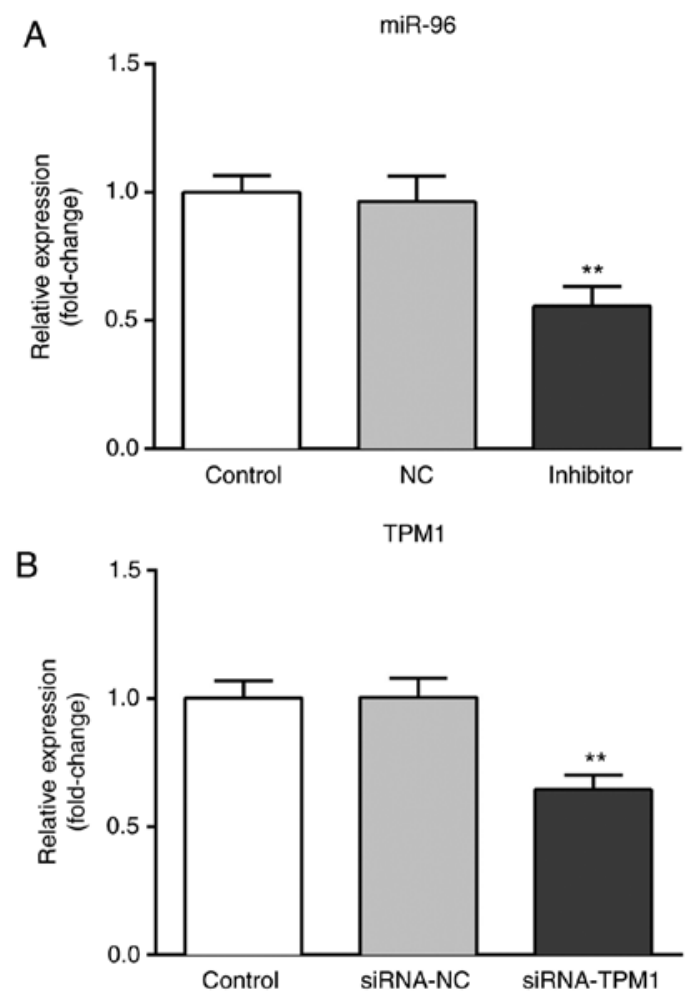

Figure 3. Transfection efficiency of miR-96 inhibitor and TPM1 siRNA. (A) The expression of miR-96 significantly decreased following transfection with miR-96 inhibitor, while there was no significant change in miR-96 expression between control and NC groups. (B) The expression of TPM1 significantly decreased following transfection with siRNA-TPM1, while there was no significant change in TPM1 expression between control and NC groups. ${ }^{* *} \mathrm{P}<0.01$ vs. NC group. miR-96, mircoRNA-96; TPM1, tropomysin 1; siRNA-TPM1, small interfering RNA targeting TPM1; NC, negative control. 

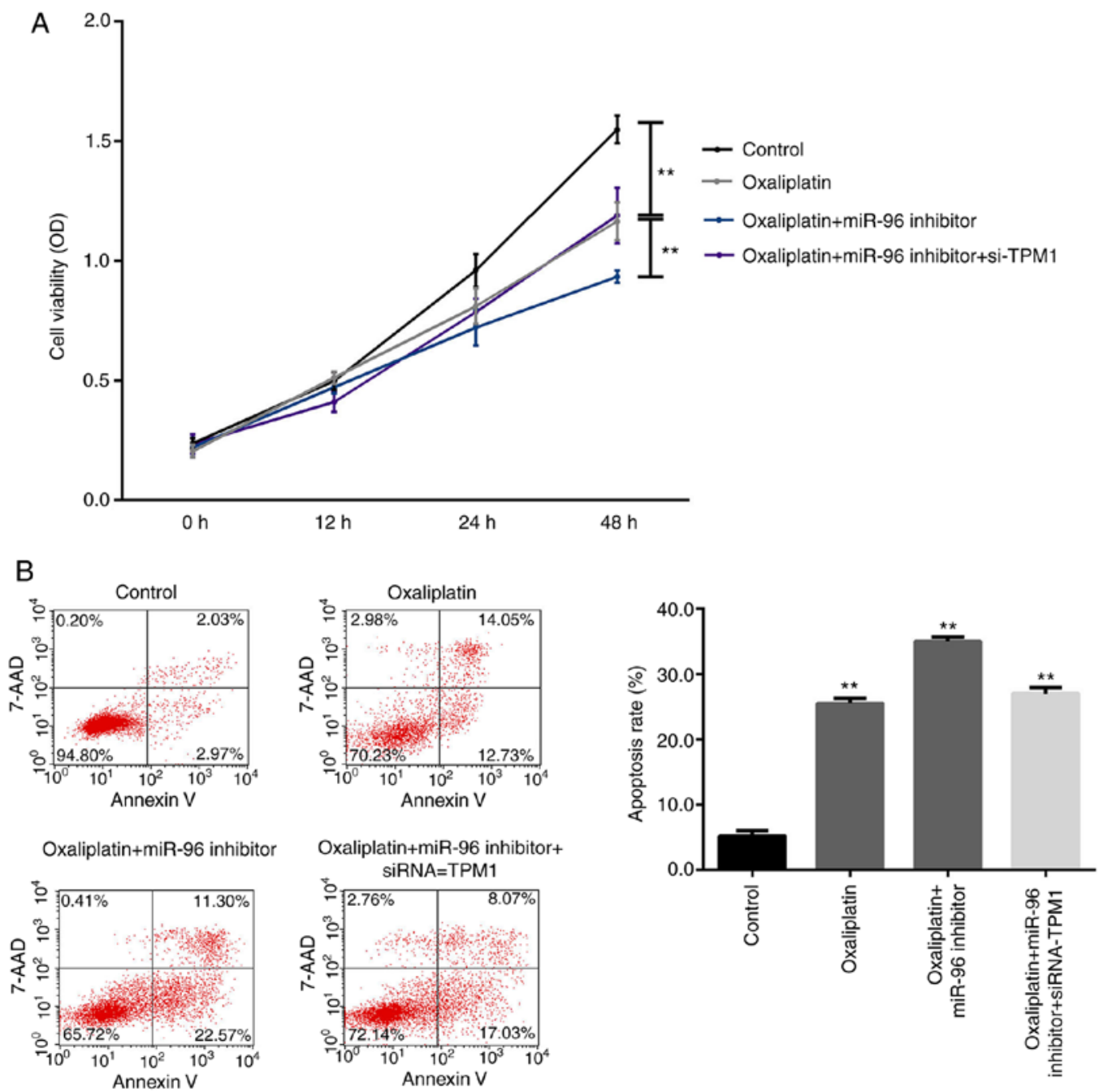

Figure 4. Downregulated miR-96 suppresses cell viability and increases the apoptopic rate of SW480. (A) Oxaliplatin significantly repressed the cell viability of SW480, which was more potent after the treatment of oxaliplatin and miR-96 inhibitor, and was then mediated by transfection with siRNA-TPM1. ${ }^{* *} \mathrm{P}<0.01$. (B) The apoptosis rate of SW480 in the oxaliplatin group was significantly higher compared with the control group. The apoptosis rate in oxaliplatin + miR-96 inhibitor group was significantly higher compared with the oxaliplatin group, which was then reversed following transfection with siRNA-TPM1. * P $<0.01$ vs. control. miR-96, mciroRNA-96; siRNA-TPM1, small interfering RNA targeting tropomysin 1; OD, optical density; 7-AAD, 7-aminoactinomycin D.

cells and promoted the progression of melanoma (23). In the present study, the potential roles of miR-96 in CRC were investigated. The results indicated that miR-96 was upregulated in CRC tissues. Previous studies also showed that the expression of miR-96 is increased in CRC tissues and that miR-96 enhanced the viability of CRC cells $(17,18)$. Therefore, it was hypothesized that knockdown of miR-96 may reverse the tumorigenic behavior of CRC cells. Considering the potential of miRNA in the chemotherapy of cancers, the present study focused on the effects of downregulation of miR-96 on the sensitivity of CRC cells to oxaliplatin.

Oxaliplatin, as a first-line anticancer agent, is a preferential therapeutic for CRC (3). In the present study, oxaliplatin suppressed the viability and promoted the apoptosis of CRC cells. Of note, miR-96 was reported to be associated with the sensitivity of cancer cells to oxaliplatin (24); however, whether miR-96 affects the chemosensitivity of CRC cells remains to be elucidated. The present study indicated that miR-96 inhibitor + oxaliplatin was more potent in inhibiting cell viability and increasing cell apoptosis of CRC cells compared with oxaliplatin alone, which demonstrated that knockdown of miR-96 enhanced the sensitivity of CRC cells to oxaliplatin. However, the underlying molecular mechanisms remained elusive.

Previous findings demonstrated that miRNAs regulate the expression of genes by targeting the 3'-UTR of their target genes (9). TPM1, predicted as a target gene of miR-96 with the online database TargetScan 7.1, has a crucial role in mediating cell proliferation and differentiation (25). Furthermore, TPM1 is downregulated in CRC, suggesting that TPM1 may act as a tumor suppressor in CRC (25). Of note, knockdown of TPM1 in miR-96 inhibitor-treated CRC cells regulated the behavior of CRC cells, including proliferation and apoptosis. Treatment with oxaliplatin + miR-96 inhibitor reduced the proliferation and promoted apoptosis of CRC cells, while this effect was inhibited by silencing of TPM1. Therefore, it may be inferred that TPM1 has positive effects on the chemosensitivity of CRC cells.

To further investigate the underlying molecular mechanisms, the expression of proliferation- and apoptosis-associated 
A

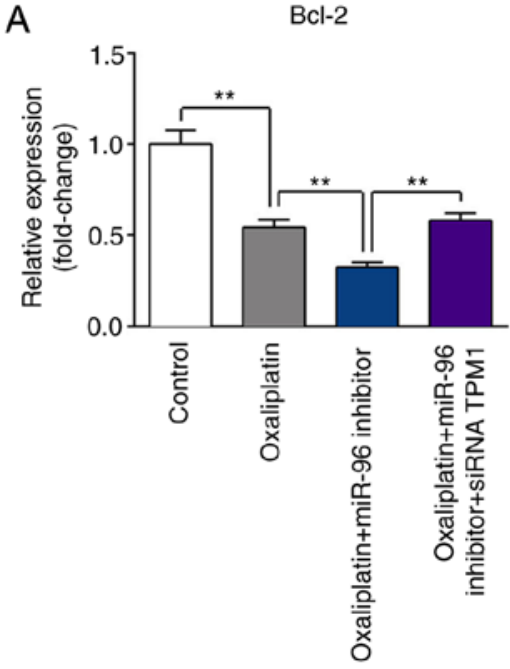

B

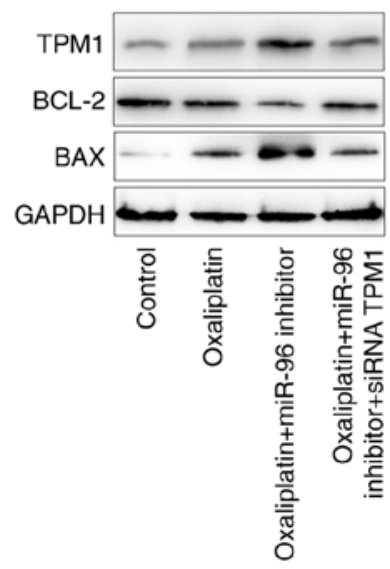

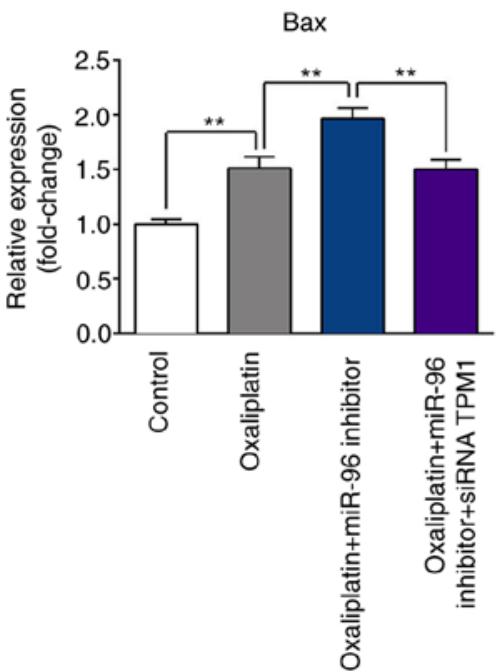

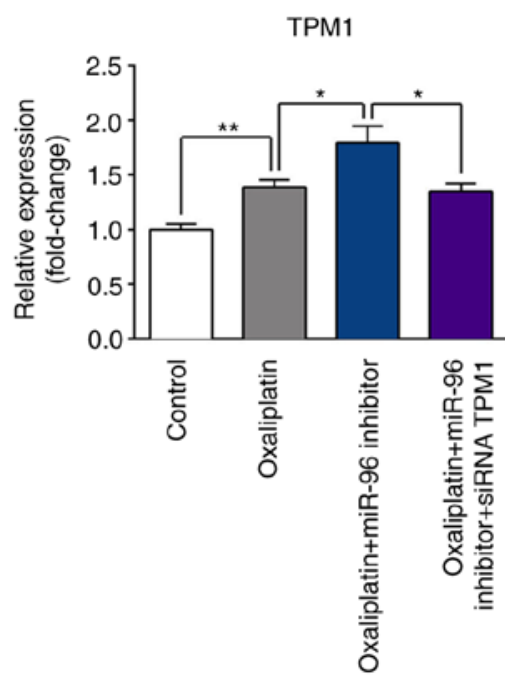

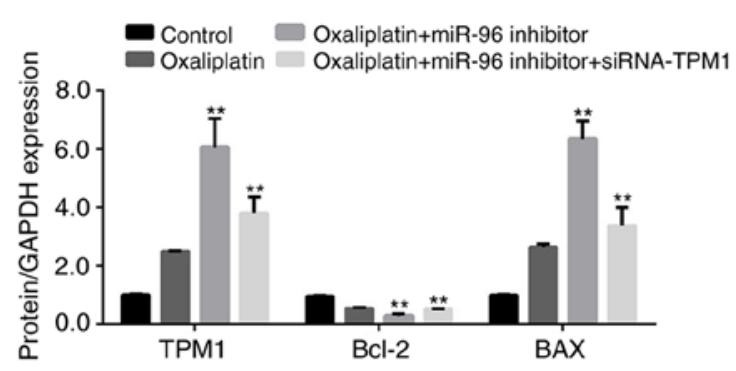

Figure 5. Low expression of miR-96 reduces the expression of Bcl-2 and increases the expression of TPM1 and BAX. (A) Oxaliplatin significantly decreased the mRNA levels of BCL-2, and increased the expression of TPM1 and BAX compared with the control group, which was more apparent in cells treated with oxaliplatin + miR-96 inhibitor. Transfection with siRNA-TPM1 attenuated this effect. ${ }^{*} \mathrm{P}<0.05$ and ${ }^{* *} \mathrm{P}<0.01$. (B) The protein expression of Bcl-2, TPM1 and BAX showed the same trends as mRNA expression. ${ }^{* *} \mathrm{P}<0.01$ vs. control. miR-96, microRNA-96; TPM1, tropomysin 1; siRNA-TPM1, small interfering RNA targeting TPM1.

factors, BAX and Bcl-2 was then examined. The results indicated that compared with oxaliplatin alone, treatment with oxaliplatin + miR-96 inhibitor was more potent in downregulating the expression of Bcl-2 and upregulating TPM1 and Bax in CRC cells, while this effect was inhibited by co-transfection of siRNA TPM1. These results suggested that suppression of miR-96 enhanced the chemosensitivity of CRC cells, which was negatively affected by siRNA TPM1.

As a limitation, the present study was limited to in vitro experimentation using cell lines. miRNAs may have various targets, or a target may be regulated by various upstream genes, which is to be further investigated in the future. Moreover, the current study only included one cell line. The roles of miR-96 should also be investigated in other CRC cells in future work.

In conclusion, the present study indicated that miR-96 was upregulated in CRC. Knockdown of miR-96 enhanced the chemosensitivity of CRC cells to oxaliplatin via targeting TPM1. These results may lay a foundation for further studies for the potential clinical application of miR-96 as a therapeutic for the treatment of CRC.

\section{Acknowledgements}

Not applicable.

\section{Funding}

No funding was received.

\section{Availability of data and materials}

The datasets used and analyzed during the present study are available from the corresponding author on reasonable request.

\section{Authors' contributions}

TG performed most experiments and statistical analysis, and revised the manuscript. PX and HM performed cell experiments. ST performed statistical analysis. JZ performed clinical experiments, literature research and wrote part of the manuscript. YZ designed the study and drafted the manuscript. All authors read and approved the final manuscript. 


\section{Ethics approval and consent to participate}

The present study was approved by the Ethics Committee of the First People's Hospital of Lianyungang. All patients provided written informed consent.

\section{Patient consent for publication}

Not applicable.

\section{Competing interests}

The authors declare that they have no competing interests.

\section{References}

1. Liebs S, Keilholz U, Kehler I, Schweiger C, Hayback J and Nonnenmacher A: Detection of mutations in circulating cell-free DNA in relation to disease stage in colorectal cancer. Cancer Med 8: 3761-3769, 2019.

2. Lei L, Zhao X, Liu S, Cao Q, Yan B and Yang J: MicroRNA-3607 inhibits the tumorigenesis of colorectal cancer by targeting DDI2 and regulating the DNA damage repair pathway. Apoptosis 24: 662-672, 2019

3. Wang Q, Wei J, Wang C, Zhang T, Huang D, Wei F, He F, Cai W, Yang P, Zeng S, et al: Gambogic acid reverses oxaliplatin resistance in colorectal cancer by increasing intracellular platinum levels. Oncol Lett 16: 2366-2372, 2018.

4. Chibaudel B, Tournigand C, Bonnetain F, Maindrault-Goebel F, Lledo G, André T, Larsen AK, Bengrine-Lefevre L, Louvet C and de Gramont A: Platinum-sensitivity in metastatic colorectal cancer: Towards a definition. Eur J Cancer 49: 3813-3820, 2013.

5. Huang CY, Chiang SF, Chen WT, Ke TW, Chen TW, You YS, Lin CY, Chao KSC and Huang CY: HMGB1 promotes ERK-mediated mitochondrial Drp1 phosphorylation for chemoresistance through RAGE in colorectal cancer. Cell Death Dis 9: $1004,2018$.

6. Ren J, Ding L, Zhang D, Shi G, Xu Q, Shen S, Wang Y, Wang T and Hou Y: Carcinoma-associated fibroblasts promote the stemness and chemoresistance of colorectal cancer by transferring exosomal lncRNA H19. Theranostics 8: 3932-3948, 2018.

7. Zhang Z, Feng L, Liu P and Duan W: ANRIL promotes chemoresistance via disturbing expression of $\mathrm{ABCC} 1$ by regulating the expression of Let-7a in colorectal cancer. Biosci Rep 38: pii: BSR20180620, 2018.

8. Gupta P, Sata TN, Yadav AK, Mishra A, Vats N, Hossain MM, Sanal MG and Venugopal SK: TGF- $\beta$ induces liver fibrosis via miRNA-181a-mediated down regulation of augmenter of liver regeneration in hepatic stellate cells. PLoS One 14: e0214534, 2019.

9. Huerta-Zavala ML, Lopez-Castillejos ES, RequenezContreras JL, Granados-Riveron JT and Aquino-Jarquin G: A single miRNA and miRNA sponge expression system for efficient modulation of miR-223 availability in mammalian cells. J Gene Med 21: e3100, 2019.

10. Wu T, Cao Y, Yang Y, Zhang X, Wang S, Xu LP and Zhang X: A three-dimensional DNA walking machine for the ultrasensitive dual-modal detection of miRNA using a fluorometer and personal glucose meter. Nanoscale 11: 11279-11284, 2019.
11. Powrozek T, Brzozowska A, Mazurek M, Mlak R, Sobieszek G and Malecka-Massalska T: Combined analysis of miRNA-181a with phase angle derived from bioelectrical impedance predicts radiotherapy-induced changes in body composition and survival of male patients with head and neck cancer. Head Neck 41: 3247-3257, 2019.

12. Vos PD, Leedman PJ, Filipovska A and Rackham O: Modulation of miRNA function by natural and synthetic RNA-binding proteins in cancer. Cell Mol Life Sci 76: 3745-3752, 2019.

13. Xie W, Sun F, Chen L and Cao X: miR-96 promotes breast cancer metastasis by suppressing MTSS1. Oncol Lett 15: 3464-3471, 2018.

14. Bao YH, Wang Y, Liu Y, Wang S and Wu B: MiR-96 expression in prostate cancer and its effect on the target gene regulation. Eur Rev Med Pharmacol Sci 21: 4548-4556, 2017.

15. Hong Y, Liang H, Uzair Ur Rehman, Wang Y, Zhang W, Zhou Y, Chen S, Yu M, Cui S, Liu M, et al: miR-96 promotes cell proliferation, migration and invasion by targeting PTPN9 in breast cancer. Sci Rep 6: 37421, 2016.

16. Wu L, Pu X, Wang Q, Cao J, Xu F, Xu LI and Li K: miR-96 induces cisplatin chemoresistance in non-small cell lung cancer cells by downregulating SAMD9. Oncol Lett 11: 945-952, 2016.

17. Rapti SM, Kontos CK, Papadopoulos IN and Scorilas A: High miR-96 levels in colorectal adenocarcinoma predict poor prognosis, particularly in patients without distant metastasis at the time of initial diagnosis. Tumour Biol 37: 11815-11824, 2016.

18. Ress AL, Stiegelbauer V, Winter E, Schwarzenbacher D, Kiesslich T, Lax S, Jahn S, Deutsch A, Bauernhofer T, Ling H, et al: MiR-96-5p influences cellular growth and is associated with poor survival in colorectal cancer patients. Mol Carcinog 54: 1442-1450, 2015.

19. Livak KJ and Schmittgen TD: Analysis of relative gene expression data using real-time quantitative PCR and the 2(-Delta Delta C(T)) method. Methods 25: 402-408, 2001.

20. Wu J, Wang F, Liu X, Zhang T, Liu F, Ge X, Mao Y and Hua D: Correlation of IDH1 and $\mathrm{B} 7 \mathrm{H} 3$ expression with prognosis of CRC patients. Eur J Surg Oncol 44: 1254-1260, 2018.

21. Fang L, Li H, Wang L, Hu J, Jin T, Wang J and Yang BB: MicroRNA-17-5p promotes chemotherapeutic drug resistance and tumour metastasis of colorectal cancer by repressing PTEN expression. Oncotarget 5: 2974-2987, 2014.

22. Zhang JR, Zhu RH and Han XP: MiR-410 affects the proliferation and apoptosis of lung cancer A549 cells through regulation of SOCS3/JAK-STAT signaling pathway. Eur Rev Med Pharmacol Sci 22: 5994-6001, 2018.

23. Jiang QQ and Liu WB: miR-25 promotes melanoma progression by regulating RNA binding motif protein 47 . Med Sci (Paris) 34 Focus issue F1: 59-65, 2018.

24. Guo Y, Pang Y, Gao X, Zhao M, Zhang X, Zhang H, Xuan B and Wang Y: MicroRNA-137 chemosensitizes colon cancer cells to the chemotherapeutic drug oxaliplatin (OXA) by targeting YBX1. Cancer Biomark 18: 1-9, 2017.

25. Mlakar V, Berginc G, Volavsek M, Stor Z, Rems M and Glavac D: Presence of activating KRAS mutations correlates significantly with expression of tumour suppressor genes DCN and TPM1 in colorectal cancer. BMC Cancer 9: 282, 2009.

This work is licensed under a Creative Commons Attribution-NonCommercial-NoDerivatives 4.0 International (CC BY-NC-ND 4.0) License. 